\title{
Stress and stressors experienced by the high-risk neonates admitted in Neonatal Intensive Care Unit and their parents: Protocol for a systematic review of available evidence from India
}

\section{Siva N}

Manipal Academy of Higher Education https://orcid.org/0000-0002-4320-7904

\section{Tenzin Phagdol}

Manipal Academy of Higher Education

Baby S Nayak ( $\square$ baby.s@manipal.edu )

Manipal Academy of Higher Education https://orcid.org/0000-0001-9659-9860

\section{Edlin Glane Mathias}

Manipal Academy of Higher Education

\section{Leslie Edward S Lewis}

Kasturba Medical College Manipal

\section{Binil Velayudhan}

Manipal Academy of Higher Education

\section{Ravi Shanker $\mathbf{N}$}

Vallabhbhai Patel Chest Institute

\section{Preethy D Souza}

UCL IOE: University College London Institute of Education

\section{Protocol}

Keywords: Stress, Stressors, High-risk neonates, Parents, Neonatal Intensive Care Unit, Systematic review, Protocol

Posted Date: November 4th, 2021

DOl: https://doi.org/10.21203/rs.3.rs-961644/v1

License: (9) (1) This work is licensed under a Creative Commons Attribution 4.0 International License. Read Full License 


\section{Abstract \\ Background}

Hospitalization of a neonate is a significant stressor for the parents as well as family members especially when a high-risk neonate is admitted to the Neonatal Intensive Care Unit (NICU) requiring continued intensive care. This prolonged physical separation from parent can have a negative psychological effect on both the neonate and the parent. These adverse health effects may last beyond hospitalization, impacting parental behavior and causing long-term emotional complications and health alteration. The neonatal period is crucial for initiating interaction and bonding between mother and child. Undue stress during this period can compromise the establishment and maintenance of a healthy mother-child bonding, which may negatively impact their long-term relationship.

\section{Methods}

The systematic review will include hospital-based observational studies, cross-sectional surveys, descriptive studies, qualitative studies, and mixed-method studies from India. Studies will be retrieved from global databases like CINAHL via EBSCO host, MEDLINE via PubMed, EMBASE, SCOPUS, Ovid, Web of Science, ProQuest Medical Library, Microsoft academic, DOAJ, and Indian databases like Indian Citation Index, J-Gate, and Neonatal Database (JCDR) using database-specific keywords. Additionally, online hand searching will be done on Indian websites of relevant institutions, women and child welfare departments, NGOs, repositories, registries, search engines, pediatric journals, and grey literature. All the primary studies reference lists will be scrutinized for additional references. Two review authors will independently screen, extract data, and critically appraise included studies. In case of any disagreements, it will be resolved in consultation with a third reviewer. Results will be reported as per the PRISMA-P guidelines.

\section{Discussion}

This systematic review will identify and highlight the stress and stressors among high-risk neonates admitted to NICU and their parents, which will guide the health care team in planning and developing comprehensive family-centered programs or interventions to reduce stress and stressors among high-risk neonates and their parents.

\section{Registration of systematic review:}

Protocol is registered in PROSPERO (International prospective register of systematic reviews), Reg ID: CRD42021272323. 


\section{Background}

One of the most traumatic situations for parents and family members is having a high-risk neonate admitted to the Neonatal Intensive Care Unit (NICU) [1, 2, 3]. The effect may last beyond hospitalization, impact parental behavior, and cause long-term emotional issues and health alterations [4]. Although 20$30 \%$ of mothers experience stress and stress disorders during neonatal hospitalizations, it is estimated that around $70 \%$ of mothers experience a higher level of stress, anxiety, and depression during the time of their high-risk neonate's admission to the NICU $[5,6]$. Some factors that exacerbate undue stress among parents are the appearance and medical condition of the neonate in NICU requiring prolonged hospitalization [7].

Common stress and stressors that parents experience during the NICU admission of their high-risk neonate include anxiety related to the neonate health condition, neonatal well-being, parenting role alteration, physical barriers, and family financial issues [8]. It also lead to social phobia, negative perception of child health, inactive maternal involvement - a moderate amount of smile, touch, and inadequate response to their neonate while caring for them [9]. These consequences would remain persistent during admission and may also endure beyond the discharge, substantially impacting mental health and functioning among parents of the high-risk neonates [10].

The following factors contribute to maternal stress significantly: (i) the anxious and fearful environment in NICU that induces parental stress likely high-risk neonate's physical traumatic appearance and behavior, visual sights, noises, equipment connected to neonates, parental role alteration, and behaviors exhibited by the health care staff. (ii) The factors which are not directly NICU environment induced that causes significant stress are: (a) Circumstantial or situational, (b) Depression and anxiety relating to personal characteristics exhibited by the parent, and (c) Distinctive assets $[11,12]$.

Approximately four percent of normal neonates and $85 \%$ of high-risk neonates are hospitalized each year into the NICU [13]. It is estimated that high-risk newborns are exposed to approximately 70 painful procedures every day in the NICU, affecting their brain function and development $[14,15]$. Invasive and non-invasive procedures in NICU will increase neonatal pain and stress. NICU bright lights, equipment noise, health care personnel noise, and touch can influence neonatal physiological responses like respiration, heart rate, blood pressure, and oxygen saturation [16].

Early adverse experiences make a neonate more sensitive to consequences of stress in child subsequent life, including an increased risk of stress-related brain development, neuromuscular development, and mental health problems $[10,17]$. Stress experienced during the neonatal period may alter the biological process, autonomic nervous system, behavior, motor function, and immune system functioning. These variations may affect the structure and function of the brain [18].

Some of the significant vital factors responsible for inducing maternal stress and anxiety are respiratory distress, vital signs, cardiac monitor alarm, and mechanical ventilator support [19]. In addition to these essential factors, high-risk neonates are frequently exposed to medical procedures, medical 
comorbidities, nursing care, and pain [20]. High-risk neonates are subjected to various situations of acute and chronic stress all through their NICU stay. These could include pain-related trauma (e.g., laboratory investigations), disturbances in the environment (e.g., excessive light and noise), and physiologic variations in their body (e.g., sepsis). Other examples include vigorous repositioning, diaper change, venipunctures, suctioning, non-invasive ventilation, and intubation. [21].

Therefore, this proposed review aims at identifying all the stress and stressors experienced by neonates admitted in the Neonatal Intensive Care Unit and their parent's stress and stressors to help guide the health care team in planning and developing comprehensive family-centered programs or interventions to reduce stress and stressors among high-risk neonates and their parents.

\section{Why Is It Important To Do This Review?}

Due to numerous adverse effects of neonatal stress and parental stress especially during their early stage of life [24, 25], many research studies were conducted to assess the stress level and understand the stressors so as to remove the cause and improve bio-physiological and psychological outcomes as well as family care. Qualitative studies emphasized that parents of high-risk neonates undergoes several negative experiences, including anxiety, stigma, guilt, depression, fear, lack of control, hopelessness, and many other emotional and psychological problems [26, 27]. Quantitative research studies have provided critical numerical data about parental stress and high-risk neonatal stress during NICU admission [3]. Systematic reviews allows a systematic assessment of stressors and causes of maternal and neonatal stress $[7,28]$. Several systematic reviews were carried out on the topic and one of the latest reviews done was on global perspective on parental stress in the neonatal intensive care unit: a meta-analytic study which reported parental role alteration as the greatest source of stress for both mothers and fathers. Mothers had higher stress levels compared to fathers [(25)]. Another systematic review assessed fathers' stress in the Neonatal Intensive Care Unit which reported that fathers find the NICU environment stressful and are more stressed than fathers of full-term, healthy infants. Their alteration in parental role, infant appearance, NICU environment, and staff communication were identified as stressors (26)]. The similar findings from these two reviews were further proved by another qualitative systematic review that explored the parental experiences in the Neonatal Intensive Care Unit. The review reported the common themes across parents' experiences were the stress of hospitalization, alteration in parenting roles and the impact of infant hospitalization on psychological health and interrupted development of a healthy parent-infant attachment (27)]. Therefore, there's global evidence on experience of parents with neonates admitted in NICU but there's no systematic reviews on stress and stressors experienced by neonates admitted in NICU. This lack of evidence can be due to challenges in assessing the stress of neonates subjectively as well as objectively. Salivary cortisol is a significant biomarker and a non-invasive method to estimate the stress levels in high-risk neonates. Newnham et al. implemented the non-invasive practical "neonatal infant stressor scale" (NISS) to measure how much stress the neonates go through in the NICU [22]. Miles developed a "Parental Stressor Scale: Neonatal Intensive Care Unit" (PSS: NICU) questionnaire to quantify the stress of mothers during high-risk newborn admission to NICU [23]. There are primary studies using these scales to assess stress in neonates. Therefore, this proposed review aims 
at assessing the stress and stressors of neonates admitted in the NICU as well as that of parents especially in India because often the evidence from the global reviews is not representative of low-and middle-countries like India due to their inaccessibility to important resources. This review will extensively search Indian resources to produce indigenized evidence that is relevant to India.

The findings of this review will inform the health policy makers, the health care organizations, associations as well as health care professionals to develop tailored interventions to minimize the stress among parents, and high-risk neonates admitted to our NICU setting. Thereby, parents can cope with negative experiences in NICU and be involved in optimal care for their high-risk neonates without any undue stress.

\section{Review questions}

What are the stress and stressors experienced by the high-risk neonates admitted to NICU?

What are the stress and stressors experienced by the parents of high-risk neonate admitted to NICU?

\section{Specific objectives}

To identify the stress experienced by the parents of high-risk neonates admitted to NICU.

To assess the stress experienced by the high-risk neonates admitted to NICU

To explore the stressors experienced by the parents of high-risk neonates admitted to NICU.

To explore the stressors experienced by the high-risk neonates admitted to NICU.

\section{Methods/design}

The systematic review was registered with the International Prospective Register of Systematic Reviews (PROSPERO) on August 2021, Reg ID: CRD42021272323. We followed the PRISMA-P (Preferred Reporting Items for Systematic Review and Meta-analysis) guidelines to describe this protocol. (Supplementary data file 1).

\section{Eligibility criteria}

\section{Types of studies}

All types of hospital-based observational studies, cross-sectional surveys, descriptive studies, qualitative studies, and mixed-method studies will be included in the review.

Time: English articles published from January 2000 to December 2021 with full text will be included.

\section{Exclusion criteria}


All experimental studies, studies not conducted in India, studies on the stress level of family members (other than parents) of high-risk neonates admitted into NICU will be excluded. Study designs such as case reports, letters, editorials, case-control, cohort studies, and narrative reviews will be excluded. Studies wherein neonates were admitted to NICU for 24 hours of observation will not be eligible. Studies of mothers with a history of diagnosed prenatal and postnatal psychiatric illness will be excluded as well.

\section{Type of participants}

This review will include high-risk neonates admitted to NICU \& their parents irrespective of the father's age, maternal age, gestational age, type of delivery, neonatal medical condition, gender, and neonatal birth weight.

\section{Type of outcome}

Stress and stressors experienced by the parents of high-risk neonates admitted to NICU.

Stress and stressors experienced by the high-risk neonates admitted to NICU.

\section{Definition of terms}

Stress: Any intrinsic or extrinsic feeling of emotional or physical tension that evokes a biological response is known as stress [38].

Stressors: A chemical or biological element, emotional consequences, environmental condition or an external stimulus that causes stress [29].

\section{Search strategy}

A comprehensive search would be conducted in Global databases like CINAHL via EBSCO host, MEDLINE via PubMed, EMBASE, SCOPUS, Ovid, Web of Science, ProQuest Medical Library, Microsoft academic, and DOAJ. Indian databases like Indian Citation Index, J-Gate, and Neonatal Database (JCDR) by utilizing the stated search keywords: Mother*, Caregiver, Caregiver*, Parent*, Female parent, Preterm baby, Low birth weight babie*, Newborn*, neonat*, Premature infant*, High-risk neonat*, high-risk newborn, "Maternal Stress", "Neonatal stress", Stress, Psychological stress, Mental stress, Depression, Stressors, Anxiety, Fear, Tension, "Neonatal Intensive Care Unit", NICU, "Neonatal Care Unit", "Neonatal critical care unit", "Observational studies", "Cross-sectional studies", "Descriptive survey”, "qualitative studies", "mixed method studies". (Appendix 1).

\section{Searching for other resources}

Online hand searching will be done on Indian websites like institutional websites, women and child welfare websites, NGOs, repositories, registries, search engines, pediatric journals, and gray literature. All primary studies reference lists will be scrutinized for additional studies. For data management, identified records will be exported to endnote $\mathrm{X} 7$.. 


\section{Data collection}

\section{Selection of studies}

Two review authors (initials SN, TP) will screen each study title and abstract the remaining search results independently. The studies will be coded to be retrieved (potentially eligible or ineligible /unclear) or not to be retrieved. All full-text articles which are eligible for inclusion will be retrieved, independently screened, and reasons recorded to exclude ineligible studies. If there is a disagreement, it will be resolved by mutual conversation or, if necessary, consultation with the third review author. (BSN). (Supplementary data file).

If numerous reports of the same study are identified, the reviewers will identify and exclude the duplicates, as well as aggregate multiple reports of the same study. The JBI critical appraisal tool will be utilized to assess the quality of studies. We will report in the PRISMA flowchart format outlining with illustrations on our process of selection and choice. (Fig.1)

\section{Data extraction and management (selection \& coding)}

A standard form will be developed and utilized for extracting the data. Before its use in the review, this data extraction form will be further piloted and thoroughly revised according to the need.

In areas where information or any data is found to be lost or lacking, each exertion will be made to get in touch with the author, or the request will be made to the author to provide added data or information. After the independent review of data extraction by the two reviewers, it will be further designated to a third reviewer for other erratic resolutions of any inconsistencies.

\section{Assessment of quality of the studies}

The studies' quality will be independently appraised by two review authors, aiming to resolve contradictions by conferencing with the third author (30)]

\section{Quantitative studies}

We will use the Joanna Briggs Institute (JBI) Critical Appraisal Checklist to identify the risk of bias and selection bias (High-risk neonates admitted in NICU and their parents).

\section{Qualitative studies}

Critical Appraisal Skills Program (CASP) checklist will be utilized to evaluate the study quality [41].

\section{Mixed methods studies}

We will use the Mixed Methods Appraisal Tool (MMAT) to identify the bias risk (32)] (33)].

\section{Deficit Outcome}


We will assess and report whether the investigators adequately or entirely address all the outcomes.

\section{Reporting of a selective outcome}

We will identify studies suggestive of reporting specific outcomes selectively.

\section{Additional sources for bias}

We would further recognize and notify that the study is clear from all the other drawbacks that tend to be a possibility of more significant risk bias.

\section{Dealing with missing data}

Researchers from the original studies will be contacted to confirm significant research characteristics and, where feasible, collect missing numerical outcome data (e.g., when a study is identified as an abstract only). If this approach is impossible and the missing data is believed to produce significant bias, the article will be removed from consideration.

\section{Data synthesis}

All included considerable studies will be presented in a descriptive and tabular form, summarizing critical features, elements concerning design areas, assessment methods, a key indicator of risk of bias in every specific study, and study outcomes.

\section{Subgroup analyses and investigation of heterogeneity}

Meta-analyses will be undertaken if there is enough homogeneity in study design and study subjects among the selected studies. If possible, sub-group analysis will be carried out based on the study's period and location.

\section{Assessment of bias conducting the systematic review}

The review will be conducted based on this protocol, and any deviations from it will be justified in a distinct section of the review.

\section{Discussion}

When a high-risk neonate is admitted to NICU, the parent and child are separated for several weeks or months. The parent-infant bond faces several obstacles in this critical care setting [35]. It is evident from decades of research that several components of the NICU atmosphere and situation are stressful for parents, especially mothers. Mothers of NICU babies had higher stress symptoms up to one year of giving birth, with clinical stress and depression symptoms reaching up to $40 \%$ in the first three months (35)]. Another study demonstrated that the smaller diameters of the bifrontal, biparietal lobes and the amount 
of brain tissue in neonates are significantly linked to increased stress. Stressor exposure indicated a smaller brain size, which may impact neonatal brain development and functioning (36)].

Parents of high-risk neonates need various emotional support in NICU, including neonatologist attention, nursing staff support, professional psychiatric assistance, and companionship with other neonatal parents and families. Parents' willingness to communicate their emotional condition with health care professionals was very varied. The responsibilities of clinicians, nurses, social workers, and psychologists in providing emotional assistance to patients and recognizing especially vulnerable parents appeared to be uncertain (37)]. The overall purpose of NICU staff is to provide an optimal care environment and establish family-centered care that promotes neonatal growth, physical, emotional connection between parents and neonates (23)].

The systematic review findings will provide insight into stress and stressors experienced by high-risk neonates admitted to NICU and their parents. It will assist the healthcare team and policymakers in adopting and developing effective interventions or programs to alleviate neonatal and parental stress during NICU admission.

\section{Strengths and limitations}

According to the best of our knowledge, this is the first systematic review assessing neonatal stress among infants admitted in NICU and include only Indian non-experimental research studies that addresses the stress and stresses experienced by high-risk newborns admitted to the NICU and their parents. To get the intended research studies, we will undertake a comprehensive and exhaustive investigation of various international databases, Indian databases, and websites; however, only articles published in English will be considered due to a shortage of resources.

\section{Abbreviations}

NICU: Neonatal Intensive Care Unit; PROSPERO: The International Prospective Register of Systematic Reviews; PRISMA-P: Preferred Reporting Items for Systematic Review and Meta-analysis - Protocol; JBI: Joanna Briggs Institute; CASP: Critical Appraisal Skills Program; MMAT: Mixed Methods Appraisal Tool; NGO: non-governmental organization; PSS: NICU: Parental Stressor Scale: Neonatal Intensive Care Unit; NISS: Neonatal Infant Stressor Scale

\section{Declarations}

\section{Acknowledgment}

The authors appreciate the technical assistance and resources provided by the Manipal Academy of Higher Education (MAHE), Manipal.

\section{Authors' contributions}


SN and TP conceived the topic. BSN and PD conceptualized the research project, its goals, and hypotheses. BSN designed the protocol and coordinated with the team to finalize the protocol. SN, TP and BSN designed the search strategies. PD has provided the inputs to write a protocol (inclusion, exclusion criteria and methodology). RS contributed to the establishing of statistical methodology of analysis. SN wrote the manuscript with the support of BSN, TP, LL and BV. Grey literature search and online journal hand search by BV and EGM. Primary and corresponding author received valid inputs from all authors. All authors read and approved the final manuscript.

\section{Funding}

None

\section{Availability of data and materials}

This systematic review's data collection has not yet commenced. We would like to initiate the search in mid-September 2021. Following that, the data and materials produced by this systematic review can be obtained from the corresponding author.

\section{Ethics approval and consent to participate}

As this is a systematic review protocol, ethical approval is not required as we will not be directly involving human participants.

\section{Consent for publication}

Not applicable

\section{Competing interest}

The authors declare that they do not have any competing interests.

\section{Authors' details}

${ }^{1}$ Ph.D. Scholar, Department of Child Health Nursing, Manipal College of Nursing, Manipal Academy of Higher Education, Manipal, India. ${ }^{2}$ Professor, \& Head, Department of Child Health Nursing, Manipal College of Nursing, Manipal Academy of Higher Education, Manipal, India. ${ }^{3}$ Professor \& Head, Department of Pediatrics, KMC, Manipal Academy of Higher Education, Manipal, India. ${ }^{4}$ Associate Professor, Department of Mental Health Nursing, Manipal College of Nursing, Manipal Academy of Higher Education, Manipal, India. ${ }^{5}$ Assistant Professor, Department of biostatistics, Vallabhbhai Patel Chest Institute, University of Delhi. ${ }^{6}$ Senior research fellow, Social Research Institute, UCL Institute of Education, University College London, UK.

\section{References}


1. Pinelli J, Saigal S, Bill Wu Y-W, Cunningham C, DiCenso A, Steele S, et al. Patterns of change in family functioning, resources, coping and parental depression in mothers and fathers of sick newborns over the first year of life. J Neonatal Nurs. 2008 Oct 1;14(5):156-65.

2. Musabirema P, Brysiewicz P, Chipps J. Parents perceptions of stress in a neonatal intensive care unit in Rwanda. Curationis. 2015 Dec 3;38(2):1499.

3. Ganguly R, Patnaik L, Sahoo J, Pattanaik S, Sahu T. Assessment of stress among parents of neonates admitted in the neonatal intensive care unit of a tertiary care hospital in Eastern India. $J$ Educ Health Promot. 2020 Oct;30:9:288.

4. Welch MG, Hofer MA, Brunelli SA, Stark RI, Andrews HF, Austin J, et al. Family nurture intervention (FNI): methods and treatment protocol of a randomized controlled trial in the NICU. BMC Pediatr. 2012 Feb 7;12(1):14.

5. Hynan MT, Steinberg Z, Baker L, Cicco R, Geller PA, Lassen S, et al. Recommendations for mental health professionals in the NICU. J Perinatol. 2015 Dec;35(Suppl 1):14-8.

6. Woodward LJ, Bora S, Clark CAC, Montgomery-Hönger A, Pritchard VE, Spencer C, et al. VERY PRETERM BIRTH: MATERNAL EXPERIENCES OF THE NEONATAL INTENSIVE CARE ENVIRONMENT. J Perinatol Off J Calif Perinat Assoc. 2014 Jul;34(7):555-61.

7. Heidari $\mathrm{H}$, Hasanpour M, Fooladi M. Stress Management among Parents of Neonates Hospitalized in NICU: A Qualitative Study. J Caring Sci. 2017 Mar 1;6(1):29-38.

8. Holditch-Davis D, Miles MS. Mothers' stories about their experiences in the neonatal intensive care unit. Neonatal Netw NN. 2000 Apr;19(3):13-21.

9. Lin Y, Xu J, Huang J, Jia Y, Zhang J, Yan C, et al. Effects of prenatal and postnatal maternal emotional stress on toddlers' cognitive and temperamental development. J Affect Disord. 2017 Jan 1;207:9-17.

10. Govindaswamy P, Laing S, Waters D, Walker K, Spence K, Badawi N. Needs and stressors of parents of term and near-term infants in the NICU: A systematic review with best practice guidelines. Early Hum Dev. 2019 Dec 1;139:104839.

11. Varma JR, Nimbalkar SM, Patel D, Phatak AG. The Level and Sources of Stress in Mothers of Infants Admitted in Neonatal Intensive Care Unit. Indian J Psychol Med. 2019 Aug;41(4):338-42.

12. Shields-Poë D, Pinelli J. Variables associated with parental stress in neonatal intensive care units. Neonatal Netw NN. 1997 Feb;16(1):29-37.

13. Simonsen KA, Anderson-Berry AL, Delair SF, Davies HD. Early-Onset Neonatal Sepsis. Clin Microbiol Rev. 2014 Jan;27(1):21-47.

14. Weber A, Harrison TM. Reducing toxic stress in the neonatal intensive care unit to improve infant outcomes. Nurs Outlook. 2019 Apr;67(2):169-89.

15. Ranger M, Chau CMY, Garg A, Woodward TS, Beg MF, Bjornson B, et al. Neonatal pain-related stress predicts cortical thickness at age 7 years in children born very preterm. PloS One. 2013;8(10):e76702. 
16. Peng N-H, Bachman J, Jenkins R, Chen C-H, Chang Y-C, Chang Y-S, et al. Relationships Between Environmental Stressors and Stress Biobehavioral Responses of Preterm Infants in NICU. J Perinat Neonatal Nurs. 2009 Dec;23(4):363-71.

17. Graham YP, Heim C, Goodman SH, Miller AH, Nemeroff CB. The effects of neonatal stress on brain development: implications for psychopathology. Dev Psychopathol. 1999;11(3):545-65.

18. Dion Nist M, Harrison TM, Steward DK. The Biological Embedding of Neonatal Stress Exposure: A Conceptual Model Describing the Mechanisms of Stress-Induced Neurodevelopmental Impairment in Preterm Infants. Res Nurs Health. 2019 Feb;42(1):61-71.

19. Szczapa T, Gadzinowska-Szczucińska J, Jopek A. Evaluation of stress responses in parents of newborns hospitalized in the Intensive Care Unit. Ginekol Pol [Internet]. 2009 [cited 2021 Jul 24];80(5). Available from: https://journals.viamedica.pl/ginekologia_polska/article/view/46613.

20. Ranger M, Chau CMY, Garg A, Woodward TS, Beg MF, Bjornson B, et al. Neonatal pain-related stress predicts cortical thickness at age 7 years in children born very preterm. PloS One. 2013;8(10):e76702.

21. Newnham CA, Inder TE, Milgrom J. Measuring preterm cumulative stressors within the NICU: the Neonatal Infant Stressor Scale. Early Hum Dev. 2009 Sep;85(9):549-55.

22. Al Maghaireh DF, Abdullah KL, Chan CM, Piaw CY, Al Kawafha MM. Systematic review of qualitative studies exploring parental experiences in the Neonatal Intensive Care Unit. J Clin Nurs. 2016 Oct;25(19-20):2745-56.

23. Feeley N, Genest C, Niela-Vilén H, Charbonneau L, Axelin A. Parents and nurses balancing parentinfant closeness and separation: a qualitative study of NICU nurses' perceptions. BMC Pediatr. 2016 Aug 20;16(1):134.

24. Chourasia N, Surianarayanan P, Adhisivam B, Vishnu Bhat B. NICU admissions and maternal stress levels. Indian J Pediatr. 2013 May;80(5):380-4.

25. Caporali C, Pisoni C, Gasparini L, Ballante E, Zecca M, Orcesi S, et al. A global perspective on parental stress in the neonatal intensive care unit: a meta-analytic study. J Perinatol. 2020 Dec;40(12):173952.

26. Prouhet PM, Gregory MR, Russell CL, Yaeger LH. Fathers' Stress in the Neonatal Intensive Care Unit: A Systematic Review. Adv Neonatal Care Off J Natl Assoc Neonatal Nurses. 2018 Apr;18(2):105-20.

27. Al Maghaireh DF, Abdullah KL, Chan CM, Piaw CY, Al Kawafha MM. Systematic review of qualitative studies exploring parental experiences in the Neonatal Intensive Care Unit. J Clin Nurs. 2016 Oct;25(19-20):2745-56.

28. Yaribeygi H, Panahi Y, Sahraei H, Johnston TP, Sahebkar A. The impact of stress on body function: A review. EXCLI J. 2017 Jul 21;16:1057-72.

29. Stressor. I Definition of Stressor by Merriam-Webster [Internet]. [cited 2021 Jul 23]. Available from: https://www.merriam-webster.com/dictionary/stressor.

30. Paré G, Kitsiou S. Chapter 9 Methods for Literature Reviews [Internet]. Handbook of eHealth Evaluation: An Evidence-based Approach [Internet]. University of Victoria; 2017 [cited 2021 Jul 24]. Available from: https://www.ncbi.nlm.nih.gov/books/NBK481583/. 
31. Long HA, French DP, Brooks JM. Optimising the value of the critical appraisal skills programme (CASP) tool for quality appraisal in qualitative evidence synthesis. Res Methods Med Health Sci. 2020 Sep;1(1)(1):31-42.

32. Pluye P. Mixed kinds of evidence: synthesis designs and critical appraisal for systematic mixed studies reviews including qualitative, quantitative and mixed methods studies. Evid Based Med. 2015 Apr;20(2):79.

33. Hong QN, Pluye P, Fàbregues S, Bartlett G, Boardman F, Cargo M, et al. Improving the content validity of the mixed methods appraisal tool: a modified e-Delphi study. J Clin Epidemiol. 2019 Jul;111:4959.e1.

34. Thomson G, Moran VH, Axelin A, Dykes F, Flacking R. Integrating a sense of coherence into the neonatal environment. BMC Pediatr. 2013 May;22(1):84. 13(.

35. Vigod SN, Villegas L, Dennis C-L, Ross LE. Prevalence and risk factors for postpartum depression among women with preterm and low-birth-weight infants: a systematic review. BJOG Int J Obstet Gynaecol. 2010 Apr;117(5):540-50.

36. Smith GC, Gutovich J, Smyser C, Pineda R, Newnham C, Tjoeng TH, et al. NICU Stress Is Associated with Brain Development in Preterm Infants. Ann Neurol. 2011 Oct;70(4):541-9.

37. Bry A, Wigert H. Psychosocial support for parents of extremely preterm infants in neonatal intensive care: a qualitative interview study. BMC Psychol. 2019 Nov;29(1):76. 7(.

\section{Supplementary}

Appendix-1 is only available as a download in the Supplemental Files section.

\section{Figures}




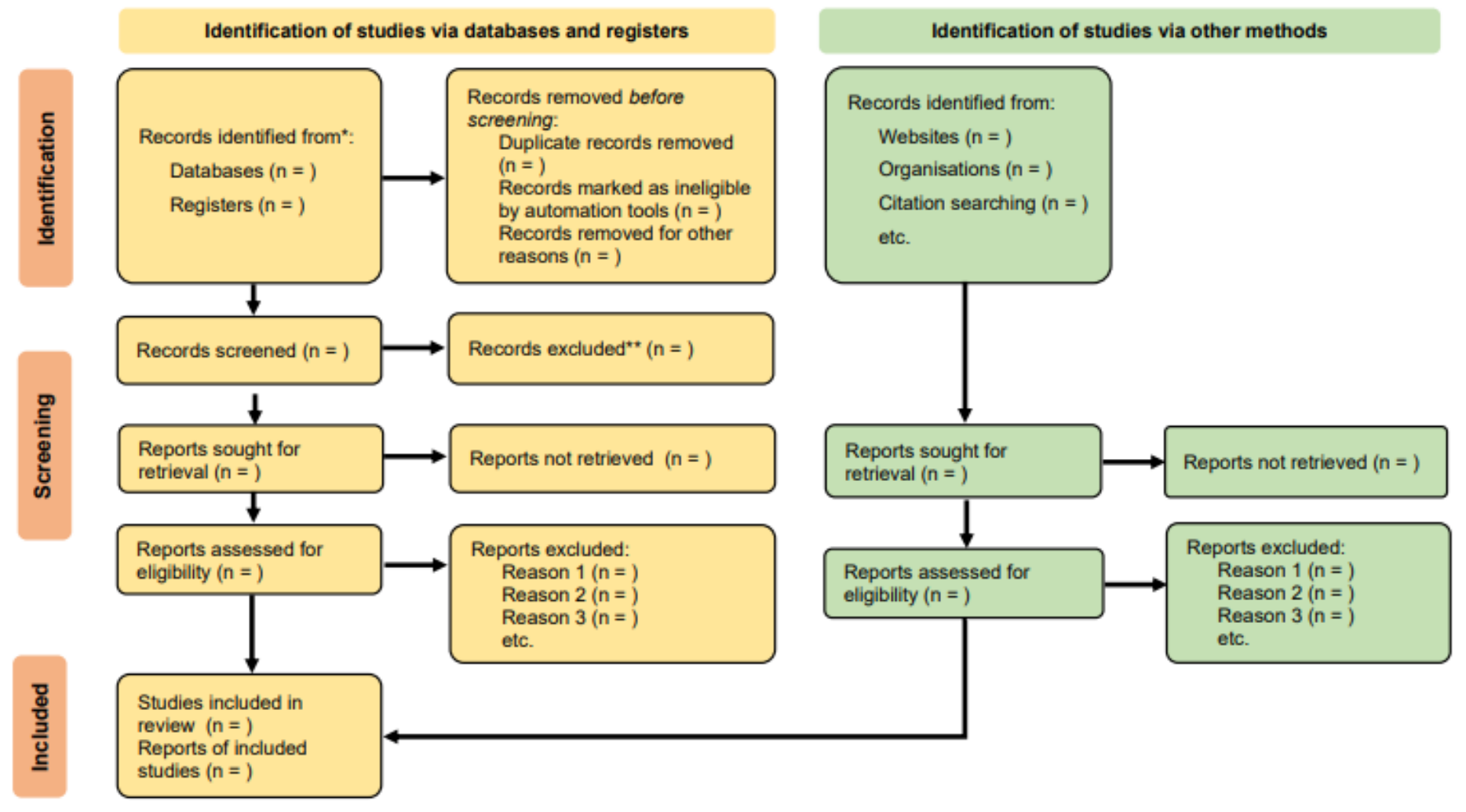

Figure 1

Flow chart of study selection

\section{Supplementary Files}

This is a list of supplementary files associated with this preprint. Click to download.

- Appendix.docx

- PRISMAPChecklist.pdf

- ScreeningChecklist.pdf 